\title{
Modeling local active sound control with remote sensors in spatially random pressure fields
}

\author{
Stephen J. Elliott and Jordan Cheer ${ }^{\mathrm{a})}$ \\ Institute of Sound and Vibration Research, University of Southampton, Southampton SO17 IBJ, \\ United Kingdom
}

(Received 20 August 2014; revised 5 February 2015; accepted 4 March 2015)

\begin{abstract}
A general formulation is presented for the optimum controller in an active system for local sound control in a spatially random primary field. The sound field in a control region is selectively attenuated using secondary sources, driven by reference sensors, all of which are potentially remote from this control region. It is shown that the optimal controller is formed of the combination of a leastsquares estimation of the primary source signals from the reference signals, and a least-squares controller driven by the primary source signals themselves. The optimum controller is also calculated using the remote microphone technique, in both the frequency and the time domains. The sound field under control is assumed to be stationary and generated by an array of primary sources, whose source strengths are specified using a spectral density matrix. This can easily be used to synthesize a diffuse primary field, if the primary sources are uncorrelated and far from the control region, but can also generate primary fields dominated by contributions from a particular direction, for example, which is shown to significantly affect the shape of the resulting zone of quiet.

(C) 2015 Acoustical Society of America. [http://dx.doi.org/10.1121/1.4916274]
\end{abstract}

[BSC]

Pages: 1936-1946

\section{INTRODUCTION}

Active sound control in enclosures works well at low frequencies, when the size of the enclosure is not too large compared with the acoustic wavelength. ${ }^{1}$ At higher frequencies, however, when global control of the sound field cannot be achieved, local active control can still be used to reduce the sound in a particular region of space. Above the Schroeder frequency ${ }^{2,3}$ the sound field can often be approximated by a diffuse field, with equal energy incident from all directions. The zone of quiet, within which the original sound level is reduced by at least $10 \mathrm{~dB}$, that can be generated by a remote secondary source around a control microphone in a diffuse field has a diameter of about one-tenth of an acoustic wavelength. ${ }^{4}$ The secondary source cannot be too remote, however, since otherwise it can increase the sound level elsewhere in the enclosure, ${ }^{5}$ and so various arrangements of active control systems with local secondary sources, close to the control region, have been investigated. ${ }^{6-9}$

The objective of the control system is often to reduce the sound level at a listener's ears. The active headphone could be seen as an ideal realization of such a local system, since the zone of quiet moves around with the position of the head. For reasons of safety and comfort, however, there has been interest in implementing such local control systems with fixed secondary source and sensor locations, particularly on headrests. $7,8,10,11$ Some investigations have assumed that knowledge of the primary waveform is available, so that feedforward methods can been used; ${ }^{7,12}$ other investigations have used feedback control, ${ }^{10,11}$ whose performance is affected by the trade-off between good acoustic performance,

\footnotetext{
a) Author to whom correspondence should be addressed. Electronic mail: j.cheer@soton.ac.uk
}

with the region of control close to the ear but relatively far from the secondary source, and good control performance, with the region of control close to the secondary source to reduce delay. ${ }^{11}$ The performance of such controllers is also affected by the spectrum of the disturbance signal and the spatial arrangement of the secondary sources, feedback sensors, and primary field. This paper presents a formulation that allows the effect of different spatial geometries to be readily calculated.

In the development of local active controllers there has also been considerable interest, for practical reasons, in monitoring the sound field within the region of control with sensors outside this region, as reviewed by Moreau et al. ${ }^{13}$ Originally a virtual microphone technique was proposed for the problem, ${ }^{13-17}$ where the primary pressure was assumed to be the same at the sensor and in the control region. Later, systems were developed using the remote microphone technique, ${ }^{13,18}$ which assumes a given transfer response between the sensor and control region. There is thus an on-going interest in the performance of active control systems that generate local zones of quiet with secondary sources and sensors outside of the region of control. This paper presents a general formulation for the calculation of the performance of such systems in a stationary primary sound field. The formulation uses spectral density matrices ${ }^{11}$ and the result is an expectation of the resulting mean square pressure in the region of control, which avoids the calculation of the multiple results that has previously been used for different realizations of the random primary field. ${ }^{4,5,15,19}$ The formulation also allows a general specification of the spatially random primary sound field, in terms of a distribution of energy from different angles, for example. It is shown that this significantly affects the results of some model calculations and that the conventional diffuse field assumption is not sufficient to 
predict the performance of local active control systems in such circumstances, as also discussed in Ref. 12.

Although the general formulation could include the effects of diffraction around finite-sized secondary sources, and the head, in the definition of the transfer responses, ${ }^{20,21}$ these effects are not included in the simulations presented here, so as to more clearly illustrate the principles of the method. The optimum control problem is first formulated in the frequency domain, to explore the geometrical limitations of control for relatively narrowband disturbances, but a time-domain formulation is also included for the calculation of causal broadband controllers. The secondary sources are assumed here to be point monopoles, and the regions of control are assumed to be far enough away from the secondary sources that the effects of the size of the secondary sources can be neglected. The simulations presented concentrate on illustrating the efficiency and the generality of the formulation. Initially the method is used to reproduce some wellknown results, such as the extent of the quiet zone in a diffuse sound field when controlled by a point source. ${ }^{19}$ Since the reference and monitor microphones are assumed to be much closer to the secondary sources than the walls of any enclosure, it is assumed that the responses between the secondary sources and these microphones are dominated by the direct sound component and hence modeled using anechoic responses. The effect of the virtual and remote microphone arrangements are calculated for this idealized situation, before the effect of a more directional random primary field is investigated. An arrangement of multiple secondary sources and reference sensors is then investigated to control an inner region in various primary sound fields. Finally, a time-domain formulation of the method is derived and some example results are discussed.

\section{DIRECT CONTROL FORMULATION}

An illustration of the physical arrangement assumed here is shown in Fig. 1. An array of primary sources, of

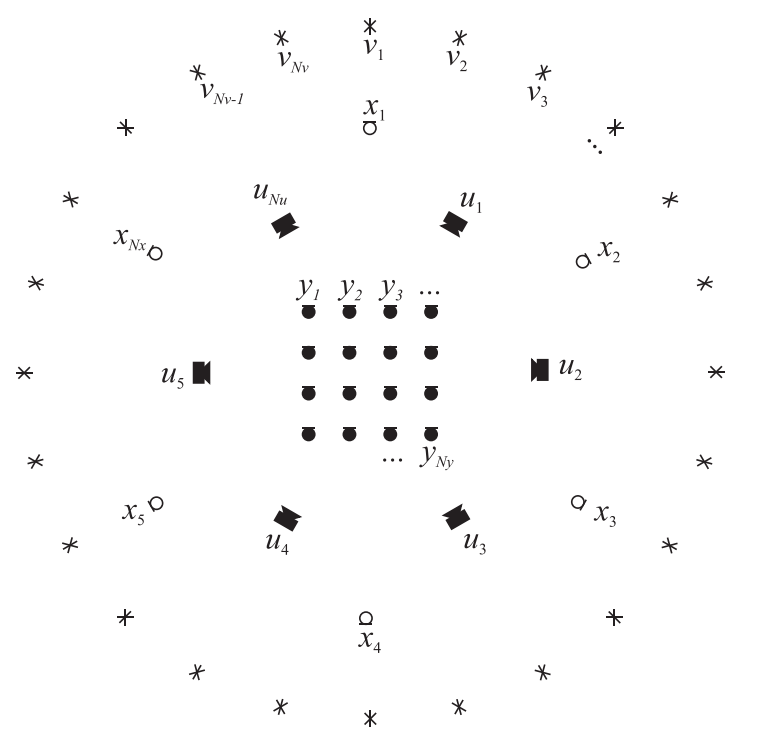

FIG. 1. Illustration of the kind of geometric arrangement assumed, in two dimensions in this case. source strengths $\mathbf{v}^{T}=\left[v_{1}, v_{2}, \ldots, v_{N_{v}}\right]$, is assumed to generate the spatially random pressure field under control. This field is detected by a set of reference sensors producing signals $\mathbf{x}^{T}=\left[x_{1}, x_{2}, \ldots, x_{N_{x}}\right]$, which are used to drive a set of secondary sources with signals $\mathbf{u}^{T}=\left[u_{1}, u_{2}, \ldots, u_{N_{u}}\right]$. The region of control is assumed to be monitored by a set of sensors with outputs $\mathbf{y}^{T}=\left[y_{1}, y_{2}, \ldots, y_{N_{y}}\right]$, some of which are used to define the cost function used in the design of the control system. Although, in the final system considered in Sec. III, the outputs from some of the more remote monitoring sensors will be assumed to be estimated from some of those closer to the secondary sources, in this section the outputs from the monitoring sensors are assumed to be known, so that the optimum acoustic performance can be calculated for a given geometry.

The arrangement is illustrated in Fig. 1 in two dimensions, but the simulations below have been conducted with the primary sources arranged on a spherical grid surrounding the local control system. It has also been assumed in this figure that the reference sensors and secondary sources are outside the region over which active control is to be implemented. The sound field in this region is measured by a dense array of monitoring sensors, so that the sound field can be visualized, even though only some of the signals from these sensors might be directly controlled. It has also been assumed in the simulations below that the transfer responses between the sources and sensors are those in a free field, although this assumption is not necessary.

In the formulation used here, the disturbances are assumed to be random, such as jet noise in aircraft or road noise in cars, for example, and can be described by their power and cross spectral densities. We will initially consider the behavior of the control system in the frequency domain. If wideband random disturbances are being controlled, an implicit assumption with the frequency domain formulation is that the matrix of control filters is causal. If this assumption does not hold, then the causality of the controller can be enforced by either using spectral factorization methods in the frequency domain ${ }^{11}$ or by formulating the controller in the time domain as a matrix of finite impulse response (FIR) filters, ${ }^{11}$ as in Sec. V. If the disturbance signals have a relatively narrowband spectrum, however, compared with the reciprocal of the delay between the reference sensors and control point, causality will not limit the performance and the frequency domain formulation can be used to predict the performance.

The relationship between the elements of the vectors of signals is represented by spectral density matrices. ${ }^{11}$ The spectral density matrix defining the primary source strengths, for example, is given by

$$
\mathbf{S}_{v v}=E\left[\mathbf{v} \mathbf{v}^{H}\right]
$$

where the superscript $H$ denotes the Hermitian, complex conjugate transpose and $E$ denotes the expectation operator. It should be noted that the dependence of all of the variables on frequency has been suppressed for notational convenience and this dependence will also be suppressed throughout the paper. The diagonal elements of $\mathbf{S}_{v v}$ are the power spectral 
densities of each individual primary source, and the offdiagonal terms are the cross spectral densities between these sources. In the simulations below it will be assumed that the primary sources are uncorrelated, so that $\mathbf{S}_{v v}$ is a diagonal matrix, although this is not necessary in the general formulation. The spectral density matrix could, for example, be used to define a spatially correlated pressure field ${ }^{22}$ or a set of original primary and secondary sources and their image sources, to approximate an enclosed sound field. ${ }^{3}$

The block diagram for the control system is illustrated in Fig. 2, where the primary sources, $\mathbf{v}$, generate the reference signals, $\mathbf{x}$, and the disturbance signals at the monitoring sensors, $\mathbf{d}$, via the matrices of transfer responses $\mathbf{R}$ and $\mathbf{P}$, respectively, and the matrix of control filters is denoted $\mathbf{W}$. The matrix of transfer responses from the secondary sources, $\mathbf{u}$, to the monitoring sensors, $\mathbf{y}$, is denoted $\mathbf{G}$, but it is assumed that any feedback from the secondary sources to the reference sensors, $\mathbf{F}$, is canceled out by a perfect model, $\hat{\mathbf{F}}$ equal to $\mathbf{F}$, within the overall controller, so that $\hat{\mathbf{x}}$ in Fig. 2 is equal to $\mathbf{x} .{ }^{11}$ The matrix of control filters, $\mathbf{W}$, which drive the secondary sources from the reference sensors, is thus entirely feedforward. The vector of complex signals at the monitoring sensors can then be written as

$$
\mathbf{y}=\mathbf{d}+\mathbf{G W} \mathbf{x}
$$

The vector of error signals to be minimized, e, is selected from the vector of signals from all the monitoring sensors, $\mathbf{y}$, via a matrix $\mathbf{A}_{e}$ so that $\mathbf{e}$ is equal to $\mathbf{A}_{e} \mathbf{y}$. The cost function to be minimized is thus

$$
J=E\left[\mathbf{e}^{H} \mathbf{e}\right]=\operatorname{trace} \mathbf{A} E\left[\mathbf{y} \mathbf{y}^{H}\right]
$$

where $\mathbf{A}$ is equal to $\mathbf{A}_{e}^{H} \mathbf{A}_{e}$ in this case and, in general, is any square Hermitian matrix.

The second form of the cost function in Eq. (3) is written in terms of the trace of the spectral density matrix ${ }^{11}$ for y. In this form, the cost function can be expanded out using Eq. (2) to give

$$
\begin{aligned}
J= & \operatorname{trace}\left[\mathbf{A} \mathbf{G W} \mathbf{S}_{x x} \mathbf{W}^{H} \mathbf{G}^{H}+\mathbf{A} \mathbf{G} \mathbf{W} \mathbf{S}_{x d}^{H}+\cdots\right. \\
& \left.+\mathbf{S}_{x d} \mathbf{W}^{H} \mathbf{G}^{H} \mathbf{A}^{H}+\mathbf{A} \mathbf{S}_{d d}\right],
\end{aligned}
$$

where the spectral density matrix for the reference signals, and the cross spectral density matrix between the output of the reference and detection sensors, are defined to be

$$
\begin{aligned}
\mathbf{S}_{x x} & =E\left[\mathbf{x x}^{H}\right], \\
\mathbf{S}_{x d} & =E\left[\mathbf{d} \mathbf{x}^{H}\right] .
\end{aligned}
$$

The properties of the trace operator, that $\operatorname{trace}(\mathbf{A}+\mathbf{B})$ is equal to $\operatorname{trace}(\mathbf{A})+\operatorname{trace}(\mathbf{B})$ and that $\operatorname{trace}(\mathbf{A B})$ is equal to $\operatorname{trace}(\mathbf{B A})$, together with the fact that $\mathbf{A}$ is Hermitian, have also been used in the formulation of the third term in the right-hand side of Eq. (4).

A generalization of the results derived in Refs. 11 and 23 then allows the optimum set of control filters to be obtained that minimize the cost function in Eq. (4), as

$$
\mathbf{W}_{\mathrm{opt}}=-\left[\mathbf{G}^{H} \mathbf{A G}\right]^{-1} \mathbf{G}^{H} \mathbf{A} \mathbf{S}_{x d} \mathbf{S}_{x x}^{-1},
$$

where it is assumed that both $\mathbf{G}^{H} \mathbf{A G}$ and $\mathbf{S}_{x x}$ are invertible.

From the block diagram shown in Fig. 2, it can be seen that $\mathbf{x}$ is equal to $\mathbf{R v}$, assuming that $\hat{\mathbf{F}}$ is equal to $\mathbf{F}$, and that $\mathbf{d}$ is equal to $\mathbf{P v}$, so that the matrices $\mathbf{S}_{x x}$ and $\mathbf{S}_{x d}$, as defined in Eq. (5), are equal to

$$
\begin{aligned}
\mathbf{S}_{x x} & =\mathbf{R S}_{v v} \mathbf{R}^{H}, \\
\mathbf{S}_{x d} & =\mathbf{P} \mathbf{S}_{v v} \mathbf{R}^{H},
\end{aligned}
$$

where $\mathbf{S}_{v v}$ is the spectral density matrix of the primary source signals, as defined in Eq. (1). The optimum matrix of control filters is thus equal to

$$
\mathbf{W}_{\mathrm{opt}}=-\left[\mathbf{G}^{H} \mathbf{A G}\right]^{-1} \mathbf{G}^{H} \mathbf{A} \mathbf{P S}{ }_{v v} \mathbf{R}^{H}\left[\mathbf{R S}_{v v} \mathbf{R}^{H}\right]^{-1},
$$

where the matrices of transfer responses $\mathbf{G}, \mathbf{R}$, and $\mathbf{P}$ are defined by the assumed geometry, $\mathbf{S}_{v v}$ is defined by the assumption of the primary field, and $\mathbf{A}$ is determined by the definition of the local field under control. It is also possible to generalize the cost function to include a control effort term, proportional to trace $\left[E\left[\mathbf{u} \mathbf{u}^{H}\right]\right]$, in addition to the control error, which has the effect of regularizing the inverse of the matrix $\mathbf{G}^{H} \mathbf{A G}$ in Eq. (10), although this was not found to be necessary in the simulations below.

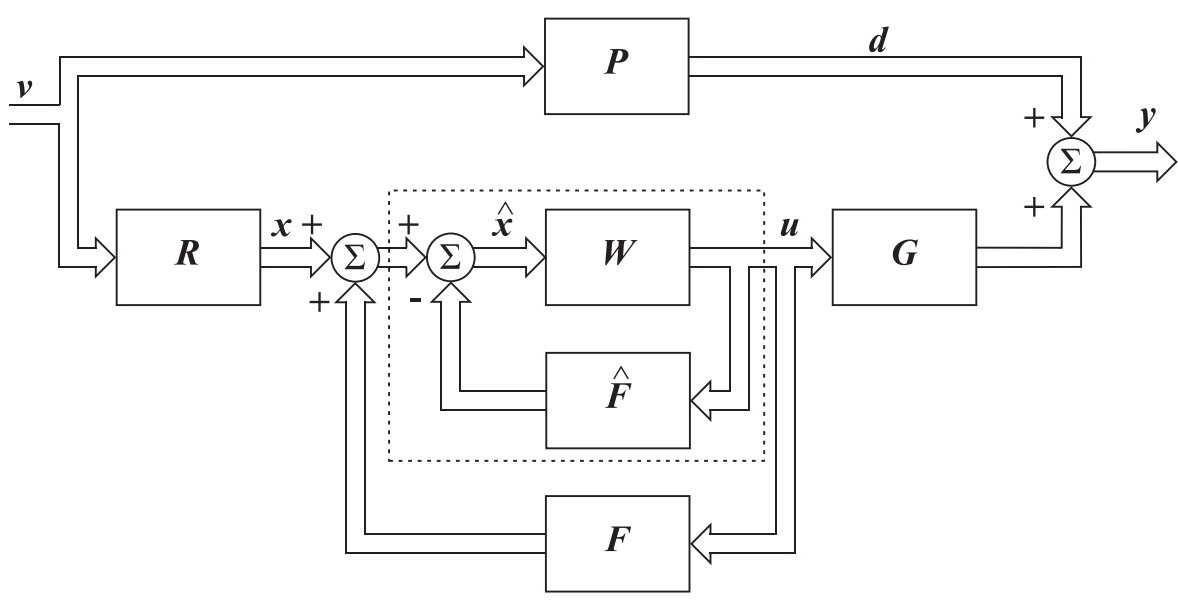

FIG. 2. The block diagram of the assumed control system. 
It is interesting to analyze the form of the optimal controller in Eq. (10), since it can be decomposed in terms of the results of two separate optimization problems. If, instead of having to control the disturbance signals with estimates of the primary source signals obtained from the reference signals, the primary source signals were directly available to drive the controller, so that

$$
\mathbf{e}=\mathbf{d}+\mathbf{G W} \mathbf{v}
$$

then the optimum least-squares controller would be equal to

$$
\mathbf{W}_{\mathrm{opt}}=-\left[\mathbf{G}^{H} \mathbf{A G}\right]^{-1} \mathbf{G}^{H} \mathbf{A} \mathbf{P} .
$$

This corresponds to the first part of Eq. (10), and can be obtained from this equation by assuming that $\mathbf{R}$ is equal to the identity matrix.

Second, we can consider the problem of optimally estimating the primary source signals, $\mathbf{v}$, from the outputs of the reference sensors, $\mathbf{x}$, via a filter $\mathbf{T}$. In this case we would minimize the cost function

$$
J_{x}=E\left[(\mathbf{v}-\mathbf{T} \mathbf{x})^{H}(\mathbf{v}-\mathbf{T} \mathbf{x})\right],
$$

resulting in the optimal filter matrix

$$
\mathbf{T}_{\mathrm{opt}}=\mathbf{S}_{v v} \mathbf{R}^{H}\left[\mathbf{R} \mathbf{S}_{v v} \mathbf{R}^{H}\right]^{-1}
$$

which forms the second part of Eq. (10). The optimal feedforward controller is thus equal to the combination of the optimum least-squares estimate of the primary source signals from the reference signals and the optimal controller driven directly by the primary source signals.

\section{REMOTE CONTROL FORMULATION}

In a number of practical active control arrangements, the signals at the error sensors are not directly observable and must be inferred from the outputs of a separate set of measurement sensors, positioned some distance from the location at which control is required. In this section the optimal controller will be derived using the remote microphone technique, ${ }^{13}$ in which an estimate of the error signals used in Sec. II, $\hat{\mathbf{e}}$, is formed from an estimate of the disturbance signals at these locations, $\hat{\mathbf{d}}_{e}$, plus the contribution due to the secondary sources operating via the plant matrix, $\mathbf{G}_{e}$, which is assumed to be known, so that

$$
\hat{\mathbf{e}}=\hat{\mathbf{d}}_{e}+\mathbf{G}_{e} \mathbf{u}
$$

where $\mathbf{G}_{e}$ is equal to $\mathbf{A}_{e} \mathbf{G}$, with $\mathbf{A}_{e}$ being defined in Sec. II.

The disturbance at the error sensors, $\mathbf{d}_{e}$, is estimated as a linear function, $\mathbf{O}$, of the disturbance at the measurement sensor positions, $\mathbf{d}_{m}$, which are also assumed to be part of the monitoring array, whose overall output is $\mathbf{y}$. In practice $\mathbf{d}_{m}$ can be obtained by subtracting a known estimate of the contribution from the secondary sources from the measured signals. We thus assume that

$$
\hat{\mathbf{d}}_{e}=\mathbf{O} \mathbf{d}_{m},
$$

where $\mathbf{d}_{m}$ can be written as $\mathbf{P}_{m} \mathbf{v}$ and the true disturbance at the error sensors, $\mathbf{d}_{e}$, can be written as $\mathbf{P}_{e} \mathbf{v}$, where $\mathbf{P}_{m}$ and $\mathbf{P}_{v}$ are subsets of the overall matrix relating the primary sources to all of the monitoring signals, $\mathbf{P}$, in Fig. 2. Using Eqs. (15) and (16) the estimated error can be written as

$$
\begin{aligned}
\hat{\mathbf{e}} & =\mathbf{O} \mathbf{d}_{m}+\mathbf{G}_{e} \mathbf{u} \\
& =\mathbf{O} \mathbf{P}_{m} \mathbf{v}+\mathbf{A}_{e} \mathbf{G W x},
\end{aligned}
$$

and the expectation of $\hat{\mathbf{e}}^{H} \hat{\mathbf{e}}$ can be minimized, using similar algebra to that used in Sec. II, if the matrix of control filters is equal to

$$
\mathbf{W}_{\mathrm{opt}(m)}=-\left[\mathbf{G}^{H} \mathbf{A} \mathbf{G}\right]^{-1} \mathbf{G}^{H} \mathbf{A}_{e}^{H} \mathbf{O} \mathbf{P}_{m} \mathbf{S}_{v v} \mathbf{R}^{H}\left[\mathbf{R} \mathbf{S}_{v v} \mathbf{R}^{H}\right]^{-1},
$$

where $\mathbf{A}$ is again equal to $\mathbf{A}_{e}^{H} \mathbf{A}_{e}$, and the subscript $(m)$ on $\mathbf{w}_{\text {opt }}$ denotes that this is the optimum controller using the measured signals.

The expectation of the squared error $\left(\mathbf{d}_{e}-\hat{\mathbf{d}}_{e}\right)$ can now be minimized to give an optimal value for the observation matrix, $\mathbf{O}$, in Eq. (16), as

$$
\mathbf{O}_{\mathrm{opt}}=\mathbf{P}_{e} \mathbf{S}_{v v} \mathbf{P}_{m}^{H}\left[\mathbf{P}_{m} \mathbf{S}_{v v} \mathbf{P}_{m}^{H}\right]^{-1},
$$

where it is assumed that the matrix $\mathbf{P}_{m} \mathbf{S}_{v v} \mathbf{P}_{m}^{H}$ is invertible. Substituting the optimal observation matrix into Eq. (19) then gives

$$
\begin{aligned}
\mathbf{W}_{\mathrm{opt}(m)}= & -\left[\mathbf{G}^{H} \mathbf{A} \mathbf{G}\right]^{-1} \mathbf{G}^{H} \mathbf{A}_{e}^{H} \mathbf{P}_{e} \mathbf{S}_{v v} \mathbf{P}_{m}^{H}\left[\mathbf{P}_{m} \mathbf{S}_{v v} \mathbf{P}_{m}^{H}\right]^{-1} \\
& \times \mathbf{P}_{m} \mathbf{S}_{v v} \mathbf{R}^{H}\left[\mathbf{R} \mathbf{S}_{v v} \mathbf{R}^{H}\right]^{-1}
\end{aligned}
$$

and it can be seen that the difference between $\mathbf{W}_{\text {opt }(m)}$ in Eq. (21) and $\mathbf{W}_{\text {opt }}$ in Eq. (10) is the factor of $\mathbf{A}_{e}^{H} \mathbf{P}_{e} \mathbf{S}_{v v} \mathbf{P}_{m}^{H}\left[\mathbf{P}_{m} \mathbf{S}_{v v} \mathbf{P}_{m}^{H}\right]^{-1} \mathbf{P}_{m}$, instead of $\mathbf{A} \mathbf{P S} \mathbf{S}_{v v}$, that occurs in the middle of the equation. The original expression for $\mathbf{W}_{\mathrm{opt}}$ in Eq. (10) is recovered if it is assumed that the measurement sensors are co-located with the error sensors, in which case $\mathbf{P}_{m}$ is equal to $\mathbf{P}_{e}$, so that $\mathbf{O}_{\text {opt }}$ is the identity matrix, and $\mathbf{P}_{e}$ is written as $\mathbf{A}_{e} \mathbf{P}$ so that $\mathbf{A}_{e}^{H} \mathbf{A}_{e} \mathbf{P}$ is equal to $\mathbf{A P}$.

\section{SIMULATIONS}

\section{A. Single secondary source in a diffuse field}

For comparison with earlier results, ${ }^{19}$ a series of simulations has initially been performed of control at a single position in a simulated diffuse primary sound field, using a single monopole secondary source. This is separated from the control position by a distance, $L$, assumed to be equal to $0.1 \mathrm{~m}$. The primary field was generated by 408 uncorrelated monopole sources uniformly distributed over a sphere of radius $1 \mathrm{~m}$. This resulted in the averaged spatial correlation function shown in Fig. 3, for the pressure in the $0.5 \mathrm{~m} \times 0.5 \mathrm{~m}$ region measured by a $51 \times 51$ grid of monitoring microphones, which is at the center of the sphere. Also shown in Fig. 3 is the $\sin (k r) / k r$ theoretical form for this spatial correlation function, where $k$ is the wavenumber and $r$ is 


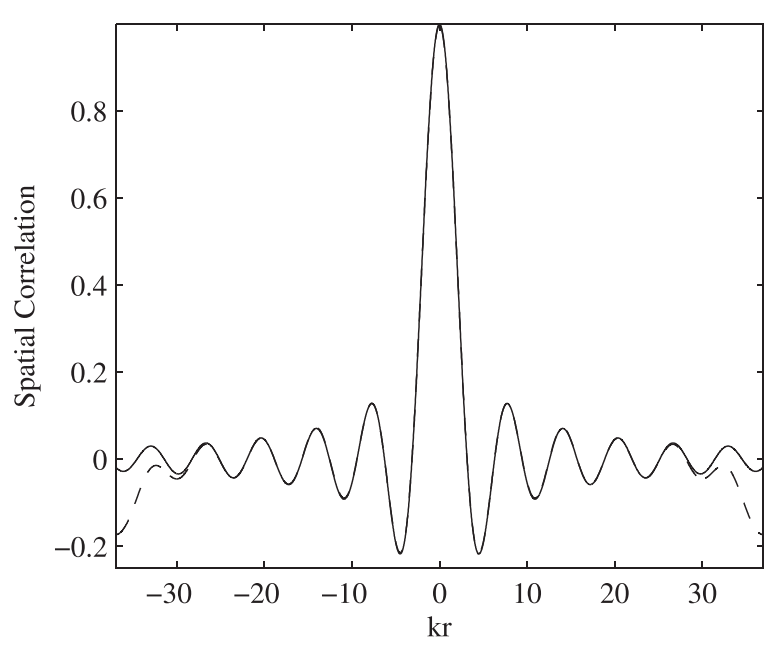

FIG. 3. Spatial correlation function for the pressure obtained for simulations of a diffuse field, dashed line, and the theoretical value, solid line.

the separation distance between the two pressures. These show a very good agreement for values of $k r$ up to about 25 , which corresponds to a frequency of around $12.5 \mathrm{kHz}$ in the simulations here, if $r$ is equal to the assumed separation, $L$, between the secondary loudspeaker and error microphone, i.e., $0.1 \mathrm{~m}$.

In these initial simulations the reference microphone is assumed to be collocated with the single error microphone at the center of the sphere of primary sources, so that the primary field at this point is perfectly measured, to be consistent with previous studies of canceling the pressure at a point. ${ }^{19}$ This is equivalent to assuming a feedback arrangement, implemented with an internal model control architecture. ${ }^{24}$ The spatial extent of the zone of quiet calculated from this simulation, within which the expectation of the primary pressure has been reduced by $10 \mathrm{~dB}$, is shown by the shaded region in Fig. 4, for various normalized excitation frequencies. The normalized excitation frequency is expressed as $k L$ where $L$ is the separation distance between the secondary source and cancellation point. These results are very similar to the results of previous calculations of the zone of quiet under these conditions, ${ }^{19}$ estimated by averaging multiple simulations, except that the zones of quiet are now more symmetrical, indicating a better estimate of the spatial extent of these zones.

Figure 5 shows the results of canceling at the same error microphone location, but with a measurement microphone positioned halfway between the error microphone at the cancellation point and the monopole secondary source, thus implementing the remote microphone method. It can be seen that the results are similar to those of Fig. 4 when the wavelength is large compared with the separation distance between the error and measurement sensor positions, so that $k L / 2$ is small compared with unity, but are somewhat degraded at higher normalized frequencies. For comparison, Fig. 6 shows the results of assuming that the primary field is the same at the error and measurement sensors, and only taking account of the difference between the deterministic secondary sound field at these points, as in the virtual microphone, arrangement. ${ }^{13,15}$ It can be seen that there is little difference between this approach and the remote
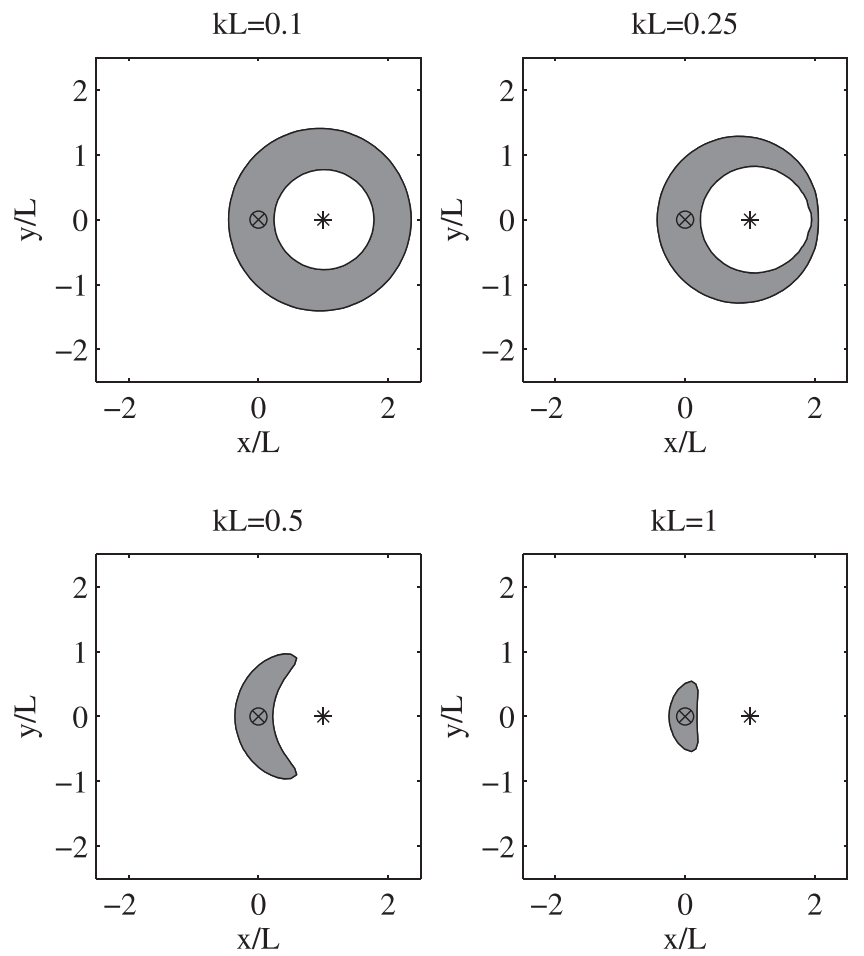

FIG. 4. The shaded regions show the zone of quiet within which the average pressure is reduced by $10 \mathrm{~dB}$ from simulations of cancellation of a diffuse field at a single point, $\times$, using an error sensor, $o$, and a single monopole secondary source, *, at four different normalized frequencies.
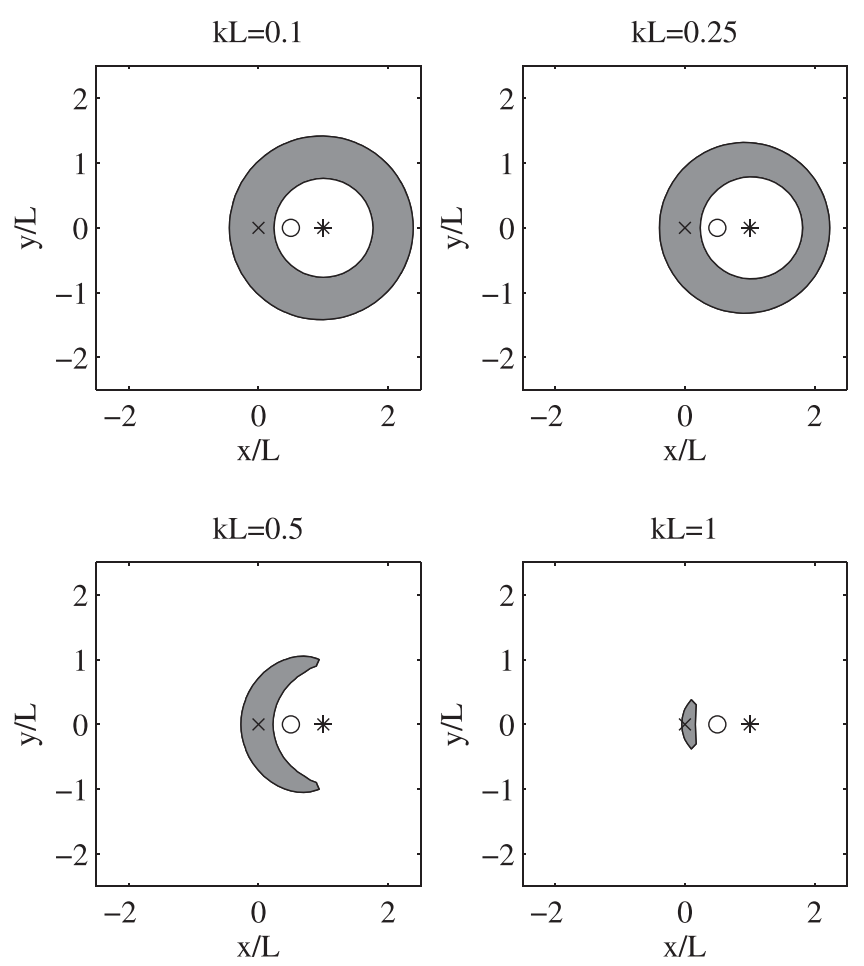

FIG. 5. The shaded regions show the zone of quiet within which the average pressure is reduced by $10 \mathrm{~dB}$ from simulations of cancellation of a diffuse field using the remote microphone method at a single error sensor point, $\times$, using a measurement sensor, o, and a single monopole secondary source, *, at four different normalized frequencies. 

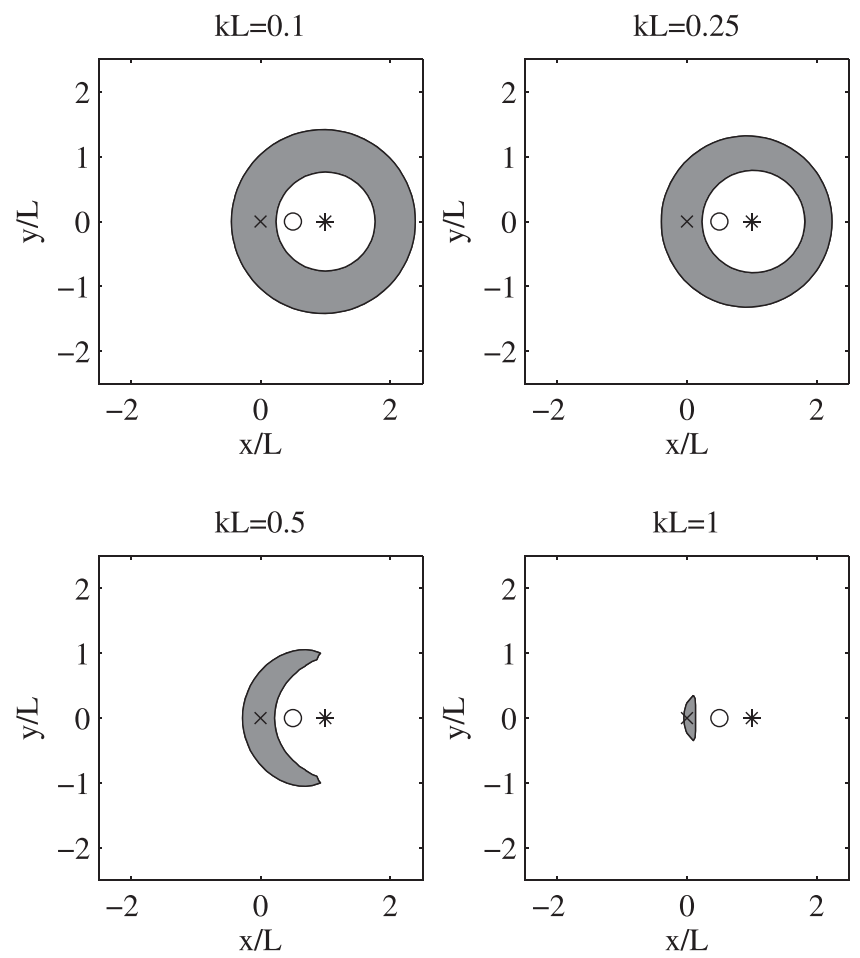

FIG. 6. The shaded regions show the zone of quiet within which the average pressure is reduced by $10 \mathrm{~dB}$ from simulations of cancellation of a diffuse field using the virtual microphone method at a single error sensor point, $\times$, using a measurement sensor, o, and a single monopole secondary source, *, at four different normalized frequencies.

microphone method in this case. It should be emphasized that all of these results were obtained with a single calculation, as opposed to the average over multiple calculations that had previously been used to calculate the performance of such systems. ${ }^{4,5,15,19}$

The performance of the three control strategies used in Figs. 4, 5, and 6 are compared at different frequencies in Fig. 7. This shows the axial extent of the $10 \mathrm{~dB}$ zone of quiet, i.e., on a line from the secondary source location to the

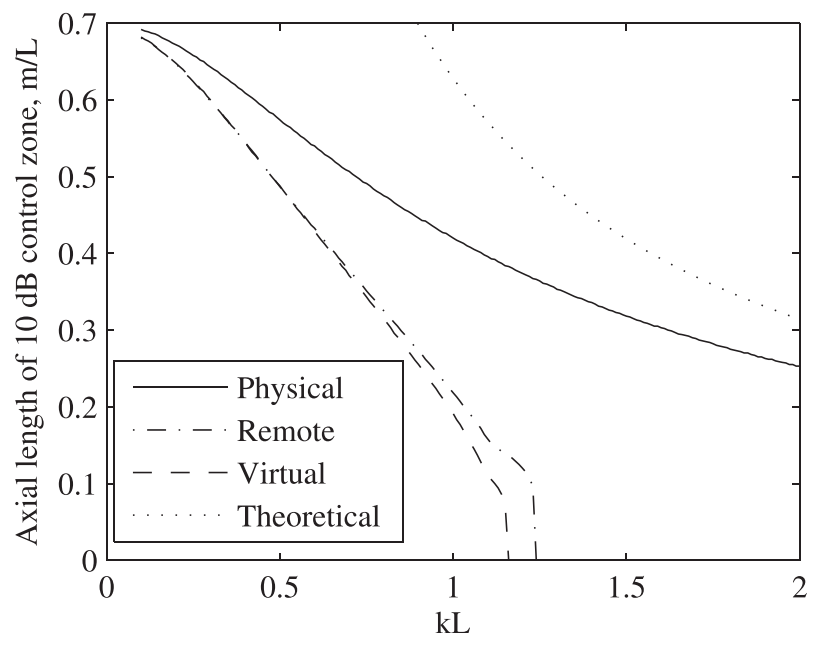

FIG. 7. Axial length of $10 \mathrm{~dB}$ zone of quiet as a function of nondimensional frequency for cancellation at a point a distance $L$ from a monopole secondary source, solid line, the use of a virtual microphone at $L /$ 2 , dashed line, the use of a remote microphone at $L / 2$, dashed-dotted line, and the theoretical $\lambda / 10$ limit for a remote secondary source, dotted line. cancellation point, as a function of normalized excitation frequency, $k L$. Not surprisingly, the case in which a physical microphone can be placed at the cancellation point always gives the best performance. The performance of the remote microphone method, in Sec. III, is degraded compared to this ideal case, but is very similar to that of the simpler virtual microphone arrangement for $k L$ less than about 0.5. Also shown in Fig. 7 are the theoretical, $\lambda / 10$, results for a remote secondary source, ${ }^{4}$ which the physical microphone approaches at higher frequencies.

\section{B. Single secondary source in a non-diffuse field}

The influence of the spatial distribution of the primary field on the shape of the zone of quiet is illustrated in Fig. 8, for the remote microphone method at a normalized excitation frequency, $k L$, equal to 0.5 . The shape of the zone of quiet for the simulations of the diffuse sound field is the same as that in Fig. 5. Also shown in this figure, however, is the shape of this zone when only 21 uncorrelated primary sources are operating, either above or to the right or to the left of the quiet zone. The zone of quiet is greatest when the primary field is mainly above the plane of the sensors and secondary source, since in this case the primary pressure field is almost uniform in the plane shown in Fig. 8, so that reductions at the control point will result in similar reductions at all positions which are a similar distance from the secondary source. When the primary field is mainly from the right, the zone of quiet is somewhat broader in the $x$-direction than that achieved with a diffuse primary field, since the phase variation of the primary field then more nearly matches that of the secondary field in this direction. Conversely, when the primary field is mainly from the left, the phase variations of the primary and secondary field match more accurately in the $y$-direction, and so the extent of the quiet zone has instead been extended in this direction.

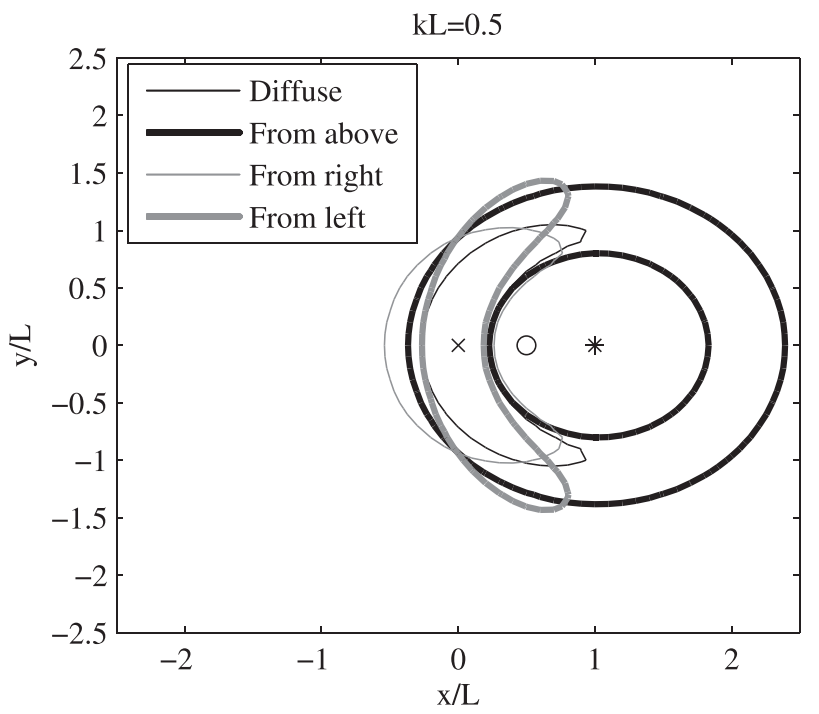

FIG. 8. The extent of the $10 \mathrm{~dB}$ zone of quiet, for a normalized excitation frequency of $k L=0.5$, when the simulated field is a diffuse, thin solid black line, mainly coming from above, thick solid black line, mainly from the right-hand side, thin solid gray line, and mainly from the left-hand side, thick solid gray line. 


\section{Multiple secondary sources in a diffuse field}

Finally, the power of the multichannel formulation is illustrated in Fig. 9, in which four monopole secondary sources, positioned at the vertices of a square of side $2 L$, are used to control the sum of the estimated mean square pressures at a $3 \times 3$ array of error microphones centrally arranged in a square of length $L$, using the remote microphone method with the signals from four measurement microphones shown by the open circles in Fig. 9. These microphones are also used to measure the reference signals in this case. The measurement sensors are located at a distance $L$ from the origin, at the midpoints of the edges of the square formed by the four secondary sources. It should be emphasized that the pressures at the error positions are estimated from the output of the measurement microphones, and no physical microphones are used in the interior of the $2 L \times 2 L$ square defined by the secondary sources, so that a listener could move their head freely in this region.

Since, in this case, the $10 \mathrm{~dB}$ zone of quiet extends over the array of monitoring microphones to a higher frequency than in the single channel case, the results in Fig. 9 are shown for the normalized frequency, $k L$, equal to $0.25,0.5$, 1 , and 1.5 , instead of $0.1,0.25,0.5$, and 1 in the single channel results above. For values of $k L$ below about 0.5 , the zone of quiet extends around the secondary sources, except within the immediate vicinity of these sources, because of their near fields. The $10 \mathrm{~dB}$ zone of quiet does not extend beyond the secondary source for normalized excitation frequencies, $k L$, above about 1, but still encloses the error sensors in this
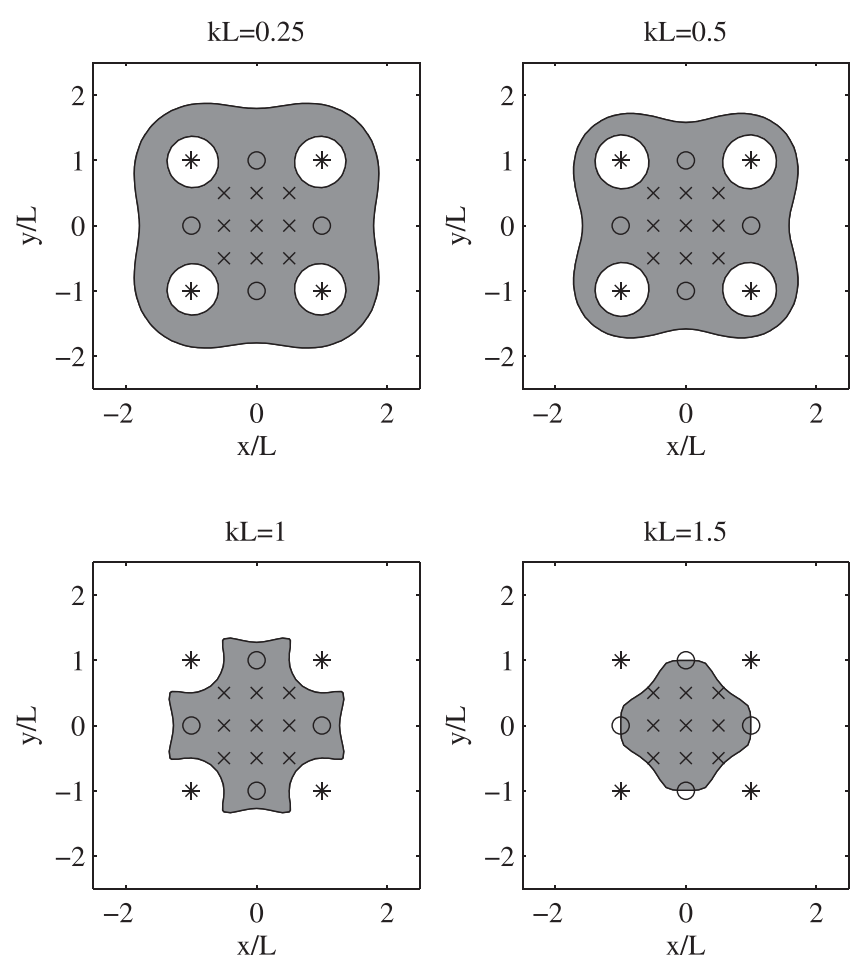

FIG. 9. Extent of the $10 \mathrm{~dB}$ zone of quiet when four secondary sources, *, are optimally driven in a diffuse field simulation to generate a zone of quiet over a $3 \times 3$ array of error microphones, $\times$, using the optimal virtual microphone techniques with four physical microphones, $\bigcirc$, at various normalized excitation frequencies. case. If $L$ was equal to $0.3 \mathrm{~m}$, for example, a normalized frequency, $k L$, of 1.5 corresponds to a physical frequency of $270 \mathrm{~Hz}$.

The results in Fig. 9 are only shown in the plane of the secondary sources and the sensors, but the diameter of the zone of quiet created with this four channel system is about $0.6 \lambda$ in this plane, which is clearly significantly more than the upper limit of $0.1 \lambda$ generated by the single channel system. ${ }^{6}$ The shape of the zone of quiet will in practice be affected by the physical size of the secondary sources and by any non-uniform directivity of the primary field. The results in Fig. 9 do indicate, however, that zones of quiet that are significantly larger than the $\lambda / 10$ single channel limit are possible using local active control systems with only four secondary sources.

\section{TIME DOMAIN FORMULATION}

The formulation of the local active noise control problem in the frequency domain, using spectral density matrices, provides an efficient method of assessing the acoustic performance for different control geometries and sound field distributions when the disturbance is narrowband. It does not, however, enforce a causality constraint on the control filters, $\mathbf{W}$, or the observation filters, $\mathbf{O}$. For broadband signals, it is generally necessary to enforce this causality constraint, which can be achieved by formulating the control problem in the time domain.

The time domain formulation can be achieved by describing the control filter and transfer responses in terms of their impulse responses and using a formulation with filtered reference signals. ${ }^{11}$ In this case the vector of sampled monitoring signals can be written as ${ }^{11}$

$$
\boldsymbol{y}(n)=\boldsymbol{d}(n)+\boldsymbol{R}(n) \boldsymbol{w}(n),
$$

where $\boldsymbol{y}(n)$ and $\boldsymbol{d}(n)$ are vectors of the $N_{y}$ sampled monitoring and disturbance signals at the $n$th sample time, and there are $N_{x}$ reference signals and $N_{u}$ secondary sources, so that $\boldsymbol{w}(n)$ is the $N_{u} N_{x} I \times 1$ vector of control filter coefficients, where each of the $N_{x} \times N_{u}$ control filters has $I$ coefficients, and $\boldsymbol{R}(n)$ is an $N_{y} \times N_{u} N_{x} I$ matrix of filtered reference signals given by

$$
\boldsymbol{R}(n)=\left[\begin{array}{ccc}
\mathbf{r}_{1}^{T}(n) \mathbf{r}_{1}^{T}(n-1) & \cdots & \mathbf{r}_{1}^{T}(n-I+1) \\
\mathbf{r}_{2}^{T}(n) \mathbf{r}_{2}^{T}(n-1) & & \\
& & \\
\mathbf{r}_{L}^{T}(n) \mathbf{r}_{L}^{T}(n-1) & \cdots & \mathbf{r}_{L}^{T}(n-I+11)
\end{array}\right],
$$

where $\mathbf{r}_{l}^{T}(n)$ is given by

$$
\mathbf{r}_{l}^{T}(n)=\left[r_{l 11}(n) r_{l 12}(n) \cdots r_{l 1 N_{x}}(n) r_{l 21}(n) \cdots r_{l N_{u} N_{x}}(n)\right]
$$

and $r_{l n_{u} n_{x}}$ is the $n_{x}$ th reference signal filtered by the plant response between the $l$ th error sensor and the $n_{u}$ th secondary source. 
If the cost function to be minimized is defined as the weighted sum of the squared error signals, which are selected from all of the monitoring signals as in Eq. (3), then using the time domain formulation, the cost function can be expressed as

$$
J=E\left[\boldsymbol{y}^{T}(n) \mathbf{A} \boldsymbol{y}(n)\right] .
$$

The vector of optimal control filter coefficients that minimizes this cost function is given by ${ }^{11}$

$$
\boldsymbol{w}_{\mathrm{opt}}=-\left\{E\left[\boldsymbol{R}^{T}(n) \mathbf{A} \boldsymbol{R}(n)\right]\right\}^{-1} E\left[\boldsymbol{R}^{T}(n) \mathbf{A} \boldsymbol{d}(n)\right] .
$$

In the context of the local active noise control system employing remote microphones, the vector of disturbance signals at the error microphones must be estimated from the vector of signals at the measurement microphones, as described in Sec. III. The matrix of optimal observation filters, $\mathbf{O}_{\text {opt }}$, is given in the frequency domain by Eq. (20), which does not enforce a constraint on the causality of the filters. Therefore, this estimation problem should also be formulated in the time domain.

The estimate of the disturbance signal at the $n_{e}$ th error sensor can be written in the time domain as

$$
\hat{d}_{n_{e}}(n)=\sum_{n_{m}=1}^{N_{m}} \sum_{j=0}^{J-1} o_{n_{e} n_{m} j} d_{n_{m}}(n-j),
$$

where $o_{n_{e} n_{m} j}$ is the $j$ th coefficient of the length $J$ FIR observation filter between the $n_{m}$ th measurement signal and the $n_{e}$ th error sensor. This can be written in matrix form as

$$
\hat{d}_{n_{e}}(n)=\mathbf{o}_{n_{e}}^{T} \boldsymbol{d}_{m}(n),
$$

where $\boldsymbol{d}_{m}(n)$ is the $J N_{m} \times 1$ vector of measurement signals and $\mathbf{o}_{n_{e}}$ is the $J N_{m} \times 1$ vector of observation filter coefficients given by

$$
\mathbf{o}_{n_{e}}=\left[\mathbf{o}_{n_{e} 1}^{T} \mathbf{o}_{n_{e} 2}^{T} \cdots \mathbf{o}_{n_{e}, N_{m}}^{T}\right]^{T}
$$

where $\mathbf{o}_{n_{e} n_{m}}$ is the $J \times 1$ vector

$$
\mathbf{o}_{n_{e} n_{m}}=\left[o_{n_{e} n_{m} 0} o_{n_{e} n_{m} 1} \cdots o_{n_{e} n_{m}(J-1)}\right]^{T} \text {. }
$$

The full vector of the $N_{e}$ estimated disturbance signals at the error sensor positions can then be written as

$$
\left[\begin{array}{c}
\hat{d}_{1}(n) \\
\hat{d}_{2}(n) \\
\vdots \\
\hat{d}_{N_{e}}(n)
\end{array}\right]=\left[\begin{array}{c}
\mathbf{o}_{1}^{T} \\
\mathbf{o}_{2}^{T} \\
\vdots \\
\mathbf{o}_{N_{e}}^{T}
\end{array}\right]\left[\begin{array}{c}
\mathbf{d}_{1}(n) \\
\mathbf{d}_{2}(n) \\
\vdots \\
\mathbf{d}_{N_{m}}(n)
\end{array}\right]
$$

so that

$$
\hat{\boldsymbol{d}}_{e}(n)=\boldsymbol{O d}_{m}(n) .
$$

As detailed in Sec. III, the optimal estimation filters can be obtained by solving the least squares problem which minimizes the error given by $\left(\boldsymbol{d}_{e}(n)-\hat{\boldsymbol{d}}_{e}(n)\right)$. Using the time domain formulation, the cost function to be minimized can be expressed as

$$
J_{O}=E\left[\left(\boldsymbol{d}_{e}(n)-\hat{\boldsymbol{d}}_{e}(n)\right)^{T}\left(\boldsymbol{d}_{e}(n)-\hat{\boldsymbol{d}}_{e}(n)\right)\right]
$$

or equivalently as

$$
J_{O}=\operatorname{trace}\left\{E\left[\left(\boldsymbol{d}_{e}(n)-\hat{\boldsymbol{d}}_{e}(n)\right)\left(\boldsymbol{d}_{e}(n)-\hat{\boldsymbol{d}}_{e}(n)\right)^{T}\right]\right\} .
$$

Substituting Eq. (32) for the estimated disturbance signal and expanding gives

$$
J_{O}=\operatorname{trace}\left\{\boldsymbol{O} \mathbf{R}_{m m} \boldsymbol{O}^{T}-\boldsymbol{O} \mathbf{R}_{e m}-\mathbf{R}_{e m}^{T} \boldsymbol{O}^{T}+E\left[\boldsymbol{d}_{e}(n) \boldsymbol{d}_{e}^{T}(n)\right]\right\},
$$

where

$$
E\left[\boldsymbol{d}_{m}(n) \boldsymbol{d}_{e}^{T}(n)\right]=\mathbf{R}_{e m}
$$

and

$$
E\left[\boldsymbol{d}_{m}(n) \boldsymbol{d}_{m}^{T}(n)\right]=\mathbf{R}_{m m},
$$

where it should be noted that these correlation matrices are not related to the transfer response $\mathbf{R}$ in Fig. 2. The matrix of causally constrained optimal estimation filter coefficients is thus given by

$$
\boldsymbol{O}_{\mathrm{opt}}=\left[\mathbf{R}_{m m}^{-1} \mathbf{R}_{e m}\right]^{T} .
$$

The full time domain solution for the optimal control filter coefficients employing remote sensors is thus given by rewriting Eq. (26) such that $\mathbf{A d}$ is equal to $\mathbf{A}_{e}^{T} \mathbf{d}_{e}$, where $\mathbf{d}_{e}=\mathbf{A}_{e} \mathbf{d}$ and $\mathbf{A}_{e}$ is now assumed to be a real matrix, and substituting $\hat{\mathbf{d}}_{e}$ in Eq. (32) for $\mathbf{d}_{e}$ with the estimation filter responses given by the optimal solution, i.e.,

$$
\mathbf{w}_{\mathrm{opt}}=\left\{E\left[\boldsymbol{R}^{T}(n) \mathbf{A} \boldsymbol{R}(n)\right]\right\}^{-1} E\left[\boldsymbol{R}^{T}(n) \mathbf{A}_{e}^{T} \boldsymbol{O}_{o p t}(n) \boldsymbol{d}_{m}(n)\right] .
$$

\section{TIME DOMAIN SIMULATIONS}

Using the optimal, causally constrained, time domain solution given by Eq. (39), the performance of the single channel system with one secondary source, one measurement microphone, and one reference microphone, in the physical arrangement shown in Fig. 10, has been evaluated as the distance between the measurement microphone and the reference microphone, and hence the distance from the reference microphone to the primary source, is varied. The

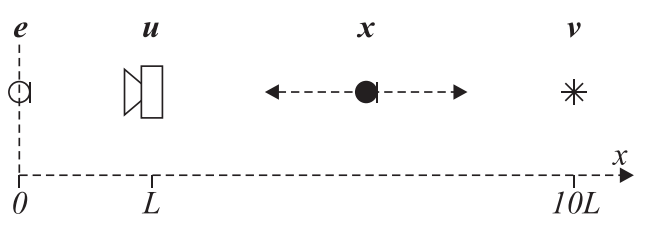

FIG. 10. Geometry of the control system with a single error sensor, single secondary source, single reference sensor, and single primary source. 
measurement microphone in these simulations is assumed to be collocated with the error microphone, located at coordinate position $(0,0,0)$, and the secondary source is located at a distance $L$ from the error sensor location in the $x$ coordinate direction, where $L$ in the simulations is taken as $0.1 \mathrm{~m}$. The primary field in this case has been generated by a single primary source located at co-ordinate position $(1,0,0)$ and this is driven by a white noise signal. To understand the effect of the causality constraint on the control system, the performance has been evaluated for both the causally constrained, time domain, solution, and the unconstrained, frequency domain, solution, as the distance between the reference microphone and the error microphone position is increased from 0 to $0.9 \mathrm{~m}$. The sample rate has been assumed to be $3.4 \mathrm{kHz}$, so that the plant response consists of a pure delay of one sample, and the length of the control filter, w, and the observation filter $\boldsymbol{O}$, have been defined as 64 coefficients. The performance of this system has been evaluated in terms of the area of the zone in which the sound pressure level is attenuated by more than $10 \mathrm{~dB}$ in the plane of control. The performance has been calculated at the normalized frequencies of $k L=0.05,0.1,0.25$, and 0.5 , and at reference microphone positions corresponding to integer values of $L$. The results of these simulations are shown in Fig. 11, with the frequency domain solutions shown by the dark lines and the time domain solutions shown by the gray lines.

From the results presented in Fig. 11, it can be seen that the performance of the frequency domain controller does not depend on the location of the reference microphone, as expected. For the causally constrained, time-domain, results, however, the performance of the controller rapidly drops off as the reference microphone is moved between the secondary source position, at a radius of $L$, and the error sensor position, at the origin. This is due to the reference signal no longer providing a time-advanced signal to the controller.

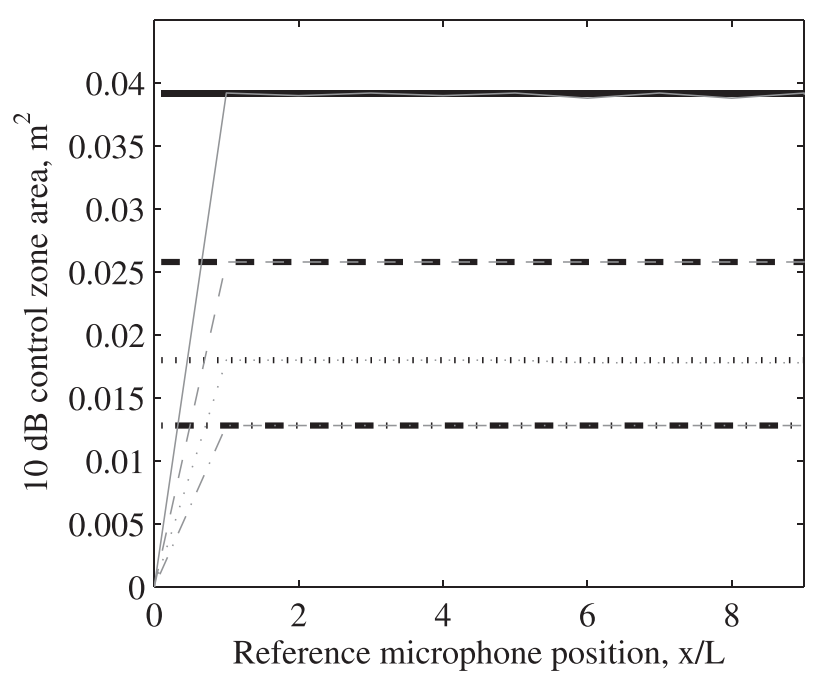

FIG. 11. The change in the area of the $10 \mathrm{~dB}$ zone of quiet for the singleinput single-output control system, controlling the field from a single primary source located at $10 \mathrm{~L}$ from the error sensor location as the position of the single reference sensor relative to the error sensor is increased from 0 to $9 L$. The thick dark lines show the frequency domain results and the thin gray lines show the time domain results. $k L=0.1$ solid line, $k L=0.25$ dashed line, $k L=0.5$ dotted line, and $k L=1$ dotted-dashed line.
That is, the wave from the primary source reaches the secondary source location before it reaches the reference microphone and, therefore, without using a negative delay time (i.e., a non-causal controller) the control system is unable to achieve control.

It is also interesting to consider the case when the primary field is produced by multiple primary sources. Using the time-domain formulation, Fig. 12, for example, shows the results for the case when six primary sources are equally distributed around the error sensor location in three dimensions, at a distance of $1 \mathrm{~m}$, as shown in Fig. 13. The secondary source and measurement microphone are located as in the previous time-domain simulations, however, in this case six reference microphones, with outputs $x_{1}$ to $x_{6}$, have been employed located along radial lines toward the positions of the six primary sources, driven by uncorrelated white noise signals, $v_{1}$ to $v_{6}$. Once again, the performance of the timedomain and frequency-domain controllers has been calculated as the reference sensors are moved from the error sensor position toward the primary source positions. From the results presented in Fig. 12, it can once again be seen that the frequency-domain predictions are not affected by the positions of the reference microphones since the six primary signals can always be perfectly recovered from the six reference signals. For the time-domain filters, however, the performance drops off as the reference sensors are moved away from the primary sources. When the reference microphones are very close to the respective primary sources, they are able to provide reference signals to the controller that are both time-advanced and coherent with the individual primary sources. However, as the reference signals are moved away from the primary sources and toward the error sensor location, both the time-advance and coherence are reduced such that all of the primary source signals cannot be recovered from the reference sensors' signals with causal filters.

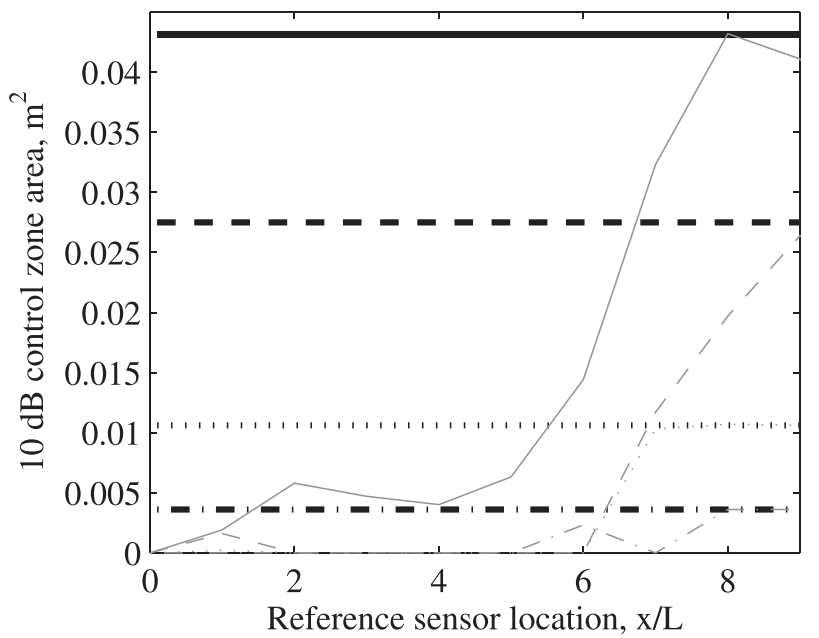

FIG. 12. The change in the area of the $10 \mathrm{~dB}$ zone of quiet for the control system shown in Fig. 13, with a single error sensor and secondary source but with six reference sensors, controlling six primary sources located at $\pm 10 \mathrm{~L}$ from the error sensor in the three coordinate directions as the distance between the six reference sensors and the error sensor is increased from 0 to $0.9 \mathrm{~L}$. The thick dark lines show the frequency domain results and the thin gray lines show the time domain results. $k L=0.1$ solid line, $k L=0.25$ dashed line, $k L=0.5$ dotted line, and $k L=1$ dotted-dashed line. 


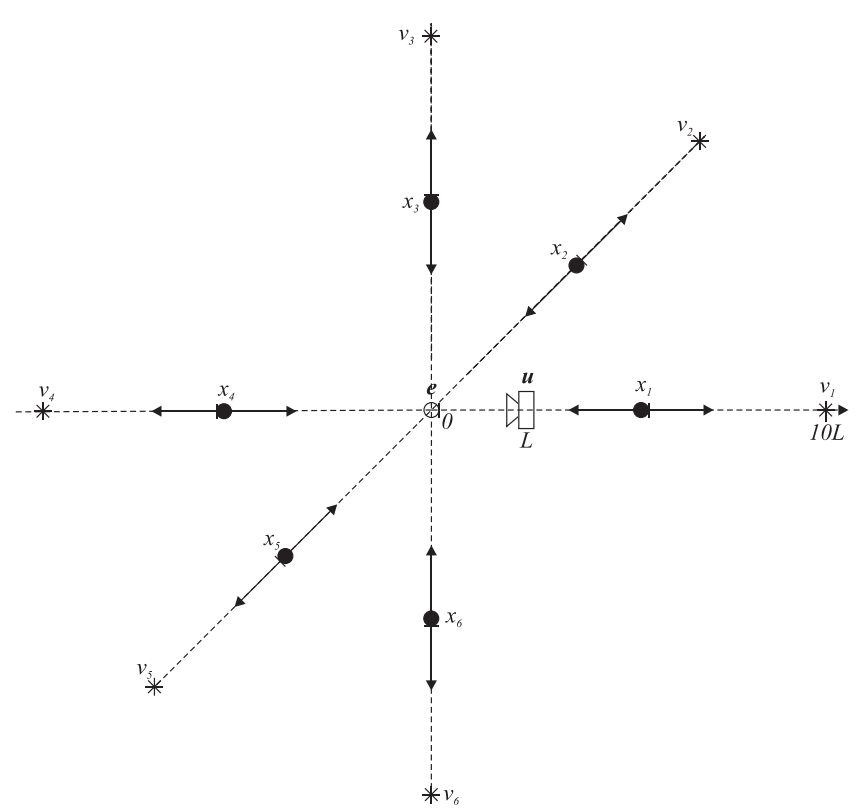

FIG. 13. Geometry of the control system with a single error sensor, single secondary source, six reference sensors, and six primary sources.

Although one reference microphone may provide a timeadvanced reference signal to the closest primary sources, it will also have components from the other primary sources that are delayed by more than the delay to the secondary source location. This means that control becomes a trade-off between canceling the component of the error signal due to the primary source closest to the reference sensor, and not enhancing the components due to the other primary sources.

\section{CONCLUSIONS}

A general formulation has been presented for the optimal least-squares solution to the local active noise control problem in a spatially random, stationary primary sound field. The field under control is assumed to be generated by an array of primary sources, specified in terms of their spectral density matrix. By assuming a uniform distribution of uncorrelated primary sources in the far field, a diffuse primary field is readily generated, but the formulation also allows more realistic primary fields to be generated, such as when it is dominated by sources in a particular direction, or in an enclosure.

The optimal least-squares controller is initially derived in the frequency domain, for the case in which the outputs of a set of error sensors can be directly measured. The form of this optimal controller is seen to be made up of two separate parts. The first part provides an optimal least-squares estimate of the primary source signals from the reference signals, and the second part is the optimal least-squares controller if the primary source signals themselves could be used to drive the controller. The problem is then considered of optimally controlling these error signals, using only the outputs from a remote set of measurement sensors. This is shown to involve an optimal form of the remote microphone method.

By way of illustration, a number of simulations that use this optimal solution are then presented, starting with local control at a point in a diffuse primary field. It is seen how the present formulation provides a single closed-form solution for the expectation of the resulting field, rather than having to average the result over many realizations of the primary field, as had been done previously.

The influence of the spatial directivity of the primary field on the shape of the zone of quiet is then explored, showing that it is important to take this directivity into account when predicting the performance of local active sound control systems. The results of a local active control system are also calculated for the case of multiple secondary sources and multiple reference signals, all outside the zone of control, which demonstrate the generality of the solution and also highlights the significant improvements that may be achieved in practice by employing a multichannel control system. Finally, a causally constrained, time domain, formulation of the control problem is formulated and the effects of the causality constraint on the control performance are calculated for the case of a control system canceling at a point operating in two different primary fields. The performance of the system in this case is dependent on both the spectral properties of the primary sources and their spatial correlation properties. The time domain formulation allows the practical performance to be predicted from experimental measurements of plant responses and sensor correlation functions.

Although the general formulation presented in this paper has been demonstrated using idealized acoustic environments, it should be emphasized that one of its strengths is its ability to rapidly assess the performance of different control geometries in a practical system using either a numerical model of the local control system or measurements in a practical arrangement. Since the presented formulation provides a closed-form solution for the expectation of the controlled sound field, it can, for example, be used to calculate the performance of different control geometries, given only the measured cross and power spectral densities of the sensor signals in the primary field and the measured transfer responses between the secondary sources and these sensors. This formulation can thus provide a powerful tool for the many calculations that need to be performed in the design of the control geometry for a local active noise control system in a practical environment.

${ }^{1}$ P. A. Nelson and S. J. Elliott, Active Control of Sound (Academic Press, London, 1992), $452 \mathrm{pp}$.

${ }^{2}$ M. R. Schroeder and K. H. Kuttruff, "On frequency response curves in rooms," J. Acoust. Soc. Am. 34, 76-80 (1962).

${ }^{3}$ A. D. Pierce, Acoustics: An Introduction to its Physical Principles and Applications (McGraw-Hill, New York, 1981), Chap. 6.

${ }^{4}$ S. J. Elliott, P. Joseph, A. J. Bullmore, and P. A. Nelson, "Active cancellation at a point in a pure tone diffuse sound field," J. Sound Vib. 120, 183-189 (1988).

${ }^{5}$ P. Joseph, S. J. Elliott, and P. A. Nelson, "Near field zones of quiet," J. Sound Vib. 172, 605-627 (1994).

${ }^{6}$ M. Miyoshi, J. Shimizu, and N. Koizumi, "On arrangements of noisecontrolled points for producing larger quiet zones with multi-point active noise control," in 23rd Inter-Noise Congress, Acoustical Society of Japan, Yokohama, Japan (1994), pp. 1299-1304.

${ }^{7}$ H. Hashimoto, K. Terai, M. Kiba, and Y. Nakama, "Active noise control for the seat audio system," in Proceedings of the 1995 International Symposium on Active Control of Sound and Vibration (Active 95), CA (1995), pp. 1279-1292.

${ }^{8}$ B. Rafaely, S. J. Elliott, and J. Garcia Bonito, "Broadband performance of an active headrest," J. Acoust. Soc. Am. 106(2), 787-793 (1999). 
${ }^{9}$ J. Guo and J. Pan, “Actively created quiet zones for broadband noise using multiple control sources and error microphones," J. Acoust. Soc. Am. 105(4), 2294-2303 (1999).

${ }^{10}$ H. F. Olson and E. G. May, "Electronic sound absorber," J. Acoust. Soc. Am. 25, 1130-1136 (1953).

${ }^{11}$ S. J. Elliott, Signal Processing for Active Control (Academic Press, London, 2001), 532 pp.

${ }^{12}$ M. Misol, C. Bloch, H. P. Monner, and M. Sinapius, "Performance of active feedforward control systems in non-ideal, synthesized diffuse sound fields," J. Acoust. Soc. Am. 135(4), 1887-1897 (2014).

${ }^{13}$ D. Moreau, B. Cazzolato, B. Zander, and C. Petersen, "A review of virtual sensing algorithms for active noise control," Algorithms 1, 69-99 (2008).

${ }^{14}$ S. J. Elliott and A. David, "A virtual microphone arrangement for local active sound control," in Proceedings of the 1st International Conference on Motion and Vibration Control, Yokohama, Japan (1992), pp. 1027-1031.

${ }^{15}$ J. Garcia-Bonito, S. J. Elliott, and C. C. Boucher, "Generation of zones of quiet using a virtual microphone arrangement," J. Acoust. Soc. Am. 101(2), 3498-3516 (1997).

${ }^{16}$ C. D. Kestell, B. S. Cazzolato, and C. H. Hansen, "Active noise control in a free field with virtual sensors," J. Acoust. Soc. Am. 109(1), 232-243 (2001).
${ }^{17}$ D. Halim, L. Cheng, and Z. Su, "Virtual sensors for active noise control in acoustic-structural coupled enclosures using structural sensing: Robust virtual sensor design," J. Acoust. Soc. Am. 129(3), 1390-1399 (2011).

${ }^{18}$ A. Roure and A. Albarrazin, "The remote microphone technique for active noise control," in Proceedings of the 1999 International Symposium on Active Control of Sound and Vibration (Active 99), FL (1999), pp. 1233-1244.

${ }^{19}$ P. Joseph, S. J. Elliott, and P. A. Nelson, "Statistical aspects of active control in harmonic enclosed sound fields," J. Sound Vib. 172(5), 629-655 (1994).

${ }^{20}$ J. Garcia-Bonito and S. J. Elliott, "Local active control of diffracted diffuse sound fields," J. Acoust. Soc. Am. 98(2), 1017-1024 (1995).

${ }^{21}$ J. Garcia-Bonito, S. J. Elliott, and M. Bonilha, "Active cancellation of pressure at a point in a pure tone diffracted diffuse sound field," J. Sound Vib. 201(1), 43-65 (1997).

${ }^{22}$ S. J. Elliott, C. Maury, and P. Gardionio, "The synthesis of spatially correlated random pressure fields," J. Acoust. Soc. Am. 117(3), 1187-1200 (2005).

${ }^{23}$ J. Minkoff, "The operation of multichannel feedforward adaptive systems," IEEE Trans. Signal Process. 45(12), 2993-3005 (1997).

${ }^{24}$ M. Morari and E. Zafiriou, Robust Process Control (Prentice-Hall, Englewood Cliffs, NJ, 1989), Chap. 12. 